\title{
MARÍA ZAMBRANO. ASPECTOS GENERALES DE SU CRÍTICA A LA RAZÓN MODERNA SECULAR Y SUS CONSECUENCIAS EN LA RELIGIOSIDAD
}

\author{
Maria Zambrano. General aspects of her critique to the modern \\ secular reason and its consequences in religiosity
}

\author{
Rodrigo Pulgar Castro*
}

\section{RESUMEN}

El estudio de Zambrano sobre la razón moderna, permite profundizar en la crisis de la cultura contemporánea, y cuya expresión absolutista de la libertad sería una de las causas del nihilismo en Occidente. Según Zambrano esta situación es manifestación de una crisis religiosa con efectos en lo político, por ello -sostendrá- la necesidad de desterrar el carácter sacrificial del poder totalitario respecto del sujeto particular, único modo de superar la trampa de la filosofía moderna que entiende la vida "instalada en el lugar del conocimiento"(El hombre y lo divino).

Palabras clave: Zambrano, secularismo, filosofía de la religión, filosofía española, intersubjetividad.

\begin{abstract}
Zambrano's study on the modern reason, allows to penetrate into the crisis of the contemporary culture, and whose absolutist expression of freedom would be one of the reasons of Western nihilism. According to Zambrano this situation is a manifestation of a religious crisis with effects in the political, for it -it will hold- the need to exile the sacrificial character of the totalitarian power respect of the particular subject, the only way
\end{abstract}

\footnotetext{
* Facultad de Humanidades y Arte, Departamento de Filosofía, Universidad de Concepción. Concepción, Chile. Correo electrónico: rpulgar@udec.cl
}

Artículo recibido el 23 de febrero de 2015. Aceptado el 10 de abril de 2016. 
of overcoming the trap of the modern philosophy that understands life "installed in the place of the knowledge" (El hombre y lo divino).

Keywords: Zambrano, Secularism, philosophy of the religion, Spanish philosophy, intersubjectivity.

\section{PROEMIO}

Si bien las lecturas críticas de María Zambrano sobre la filosofía moderna es un tema recurrente ya en textos de los años 1928 y 1930, su advertencia, y su claridad, se visualizan principalmente en dos textos: El hombre y lo divino y Persona y democracia; en ambos la filósofa profundiza según la conclusión a la cual llega Gómez Blesa en: "la angustiosa crisis de la cultura contemporánea, atisbando en la absolutización de la libertad que lleva a cabo la filosofía moderna una de las causas fundamentales del nihilismo en Occidente" (2001: 25). En ambos textos Zambrano refiere de modo principal a una crisis en el sujeto. La explicación está puesta en el hecho que en el sujeto encuentra la palpación de sus efectos. Es -si se quiere- su campo de prueba.

En términos generales, en El hombre y lo divino la crisis se tematiza como religiosa, a causa que está marcada por un ocultamiento de la piedad, entendida esta como:

la forma primeramente accesible de lo religioso, la toma de contacto, indefinible, pues toda religión comienza por lo inefable y acaba en ello, pues lo que de ella puede ser revelado en palabras es una parte mínima de todo lo que nos ofrece. Y esto inefable es, sin embargo, el fundamento de la palabra, de que haya cosas y nombres para las cosas, pues más bien se parece a un espacio, a un espacio vital donde vivimos, nos movemos y llegamos a ser (Zambrano, 2003: 52).

De suyo, así comprendida la piedad como virtud, entonces es ella la que permite identificar aquello que suscita actos de devoción y cuya matriz de sentido se encuentra en lo que se suele calificar -dentro del espectro teórico de Zambranocomo lo sacro, y cuya manifestación es el amor que se siente hacia Dios o, y no en pocas ocasiones, acciones impulsadas por el amor que:

Descubre la realidad y la inanidad de las cosas, el que descubre el no-ser y aún la nada. Y todo lo que lleva en sí una brizna de este amor descubre algún día el vacío de las cosas y en ellas, porque toda cosa y todo ser que conocemos aspira a más de lo que realmente es. Y el que ama se fija en esta aspiración, en esta realidad no lograda, en esta entelequia aún no sida y al amarla la arrastra desde el no-ser a un género de realidad que parece total un instante y que luego se oculta y aún se desvanece. Y así el amor hace transitar, ir y venir entre las zonas antagónicas de la realidad, se adentra en ellas y descubre su no-ser, sus infiernos (Zambrano, 1983: 273). 
Si en El hombre y lo divino es lo religioso, en Persona y democracia la reflexión sobre la crisis se hace desde una óptica política. Esta óptica se desenvuelve en un contexto interpretativo que, por sus características socio-seculares, la lleva a señalar la necesidad de desterrar el carácter sacrificial del poder totalitario (estatal en sentido práctico) respecto del sujeto particular. A los dos textos fundamentales se suma La España de Galdós, escrito en donde Zambrano ingresa un tercer nivel hermenéutico al estudio. Lo característico de este tercer nivel, es que ahí se despliega la razón mediadora o piadosa; la cual se asume como antecedente de la llamada razón poética entendida en su desarrollo como un método. Ya en propiedad de este instrumento epistémico, Zambrano se propone recuperar para la filosofía el thelos de la figura humana; situación posible puesto que la razón poética revela, y merced al acto en cuanto genuinamente poético, situaciones que reflejan la vida humana enganchada en aspectos que la mera razón moderna cartesiana no observa, y no las ve por la clausura de la órbita de sentido (el religioso-metafísico), pues como razón derechamente instrumental, se limita su episteme a aspectos positivos o, derechamente, analíticos. Asunto que ya Unamuno había advertido: “SSaber para saber! ¡La verdad por la verdad! Eso es inhumano” (1983: 83), y más adelante al mencionar a Descartes y sus contradicciones:

Lo malo del discurso del método de Descartes no es la duda previa metódica; no que empezara queriendo dudar de todo, lo cual no es más que un mero artificio; es que quiso empezar prescindiendo de sí mismo, del hombre real, de carne y hueso, del que no quiere morirse, para ser un mero pensador, esto es, una abstracción. Pero el hombre real volvió y se le metió en la filosofía (1983: 88).

La hipótesis de Zambrano sobre la posibilidad de recuperación del sentido humano por superación de la razón cartesiana, es plausible explicar desde un postulado crítico que, en lo medular, asume el hecho de una figura humana inserta en la órbita de la filosofía moderna, vale decir, la existencia "instalada en el lugar del conocimiento" (Zambrano, 1993); asunto que le permite concluir en un inevitable dato epistemológico; dato que interviene como cláusula del sentido de la vida ahora, por tanto, "al propio tiempo sometida a él y deificada" (Zambrano, 1993).

Asumiendo la crítica de Zambrano sobre la razón moderna que según su óptica objetiva la existencia al puro conocer racional, nos proponemos descubrir tres aspectos de la crítica suya a la razón moderna. Son tres aspectos que en términos preliminares se ven simultáneos. Este carácter sincrónico de elementos de la razón moderna es, precisamente, lo que da sustento a la crítica. Mas no se trata de quedar en ella atrapado. Por el contrario, tiene valor como ejercicio de salida a la crisis; crisis en el sujeto; crisis nacida por el imperio absoluto de la razón que se posiciona como criterio único de verdad. 
En lo sustantivo hay aspectos de su postulado que hay que considerar para entender el sentido de la crítica razón cartesiana. Dentro de estos aspectos lo principal son aquellas características propias del sujeto moderno. Nos referimos a aquel tipo humano que ve en la razón el artículo que define su esencia; el cual, a decir de Zambrano, cree: "en la razón como único medio de relacionarse con la realidad -razón discursiva o intuición intelectual-"(Zambrano, 1993: 1 y 2). Con entender este fenómeno, ya es posible dar cuenta del valor de la crítica a la razón moderna. De suyo, resulta este ser el único modo de lograr una adecuada comprensión -aunque resulte paradojal tal afirmación- sobre la secularización y su efecto en el campo de la religiosidad.

Para cumplir con lo anterior y, por tanto, resolver el sentido de la crítica, en la arquitectura de este trabajo hay presente dos ejes interpretativos: 1) La razón secular y 2) La crítica a la subjetivación del sujeto cartesiano que deriva en la clausura del sujeto moderno. Ambos ejes son puestos al servicio de lograr comprender de mejor manera el sentido de una filosofía que se propone resolver la sacralidad como experiencia constitutiva del ser persona, pues en ello radica el valor -sostendría Zambrano- de su propia humanidad.

\section{"DIOS COMO HORIZONTE DE SENTIDO"}

\section{Zambrano escribe en Hacia un saber sobre el alma que:}

hemos de dejar apuntado que lo que el hombre moviliza para engendrar la objetividad es lo religioso, como lo que hay en la base y fundamento de todo nuestro apego a la realidad y a las transformaciones que la hacemos padecer para crear nuestro mundo (1989: 99).

El texto citado denota su preocupación por aclarar que las notas que determinan la actualidad de lo religioso es la figura de Dios como horizonte de sentido, y que en su carácter de actualidad, permite que el sujeto se apropie de un habitad medianamente adecuado a lo que es su propio talante y, por consecuencia, del sentido de la vida como de la muerte. Empero, más allá de comprender que Dios para el sujeto es aquel significante esencial, queda patente que esta misma situación depende tanto de la variabilidad del tiempo como del habitad que se ocupe. Sin ambas condiciones presentes no hay traducción histórica de Dios, pues no hay condición práctica para su visualización, ya que no está la experiencia de Dios recogida en la vida práctica. Es en este contexto de interpretación que, por mor del valor del tiempo como el del espacio, nos encontramos de cara con el tiempo secular. Tiempo cuya característica en sus efectos (al menos inicial), es que se torna dificultad 
objetiva para el propósito de lograr una plena comprensión del sentido religioso, a raíz que la arquitectura vital del fenómeno que idéntica lo secular, genera una actitud epistémica en el sujeto que -y gracias a aquello- sufre permanentemente la contradicción entre una pretensión interpretativa religiosa y una razón que, desde su propia auto-limitación, lo lleva a liar con una realidad que ontológicamente sobrepasa a la razón misma. Esta percepción se acentúa al momento que el sujeto permanece en un ambiente cultural en donde: "La situación de secularización del pensamiento, la conciencia y la cultura no ha eliminado ninguna de las dimensiones e intenciones de apertura al Absoluto constituyentes de la persona" (Velasco, J.M., 2006, 194). De hecho, la extrapolación del fenómeno cultural bajo las condiciones de compleja relación tenida en la actualidad con lo religioso -algo por demás muy propio de la edad moderna en adelante (al menos en occidente y desde occidente como punto hermenéutico)-, claramente le otorga a la relación Dios-persona una categoría moral (Cortina, 1986: 71 y ss), pues a pesar del avance de lo secular, los dilemas a los que se enfrenta la conciencia particular siguen teniendo el componente de fe y de razón. Situación que ciertamente otorga un grado de dificultad comprensiva al sujeto cuando debe enfrentar la necesidad de discernir entre distintas opciones éticas. Lo dicho ciertamente va asociado al cuestionamiento proveniente de los elementos supuestos en el espacio secular-imperio de la razón, distanciamiento con la formas de vida religiosa, autonomía del orden temporal respecto de la orientación-normativa ética religiosa-eclesial, entre otros-, puesto que son elementos que debe soportar y conducir el sujeto discerniente en el día a día.

Pues bien, ante el fenómeno secular que cuestiona las variables éticas que nacen de la relación Dios-persona, se puede guardar silencio evitando la pregunta sobre lo sacro (su significante y sus efectos), pero también es posible enfrentarse a la pregunta, pues se asume su incidencia en el modo ético de existir. Un ejemplo de la segunda posibilidad es María Zambrano. Para entender por qué acepta la filósofa el desafío de resolver el sentido de lo sacro para el sujeto moderno, hay que buscar en el hecho que las claves fundamentales de su pensamiento son en general: "la necesidad de lo 'divino' en la realización del hombre; el estudio del problema de Dios como realidad en el hombre y desde el hombre (Cobos Navidad, 1998: 163). Es decir: el significado constituyente de la experiencia humana de Dios.

El asunto que las claves aparecen coherentes con aquel propósito filosófico que persigue resolver el significante de lo religioso como experiencia humana. De suyo, la clave interpretativa sobre su sentido cristaliza en el postulado de la esperanza. Mas y paradójicamente, la esperanza convive en el mismo espacio con el drama de la existencia. Y es esto lo que, según Zambrano, la filosofía debe asumir, pues ahí se explica su origen. Asunto explicado en el contexto de lo religioso y con los efectos que esto tiene en el nacimiento de la filosofía asociado al drama de la necesidad de tener respuesta: 
Sobre esta escondida fuerza religiosa, sobre esta esperanza que engendra nuestras creencias, creencias en que se afirma un orden del mundo, en que la realidad obscura ha adquirido transparencia, permanencia y sentido, surge la filosofía. Y la filosofía es razón, lo fue al menos en su comienzo. Y este es el drama (Zambrano, 1989: 99).

En sentido estricto, deberá entenderse por drama una especie de advertencia hecha a la filosofía misma que, en exceso de razón, puede llevar a la desesperanza de sentido.

\section{"EL EFECTO DE LA RAZÓN MODERNA"}

Zambrano, apoyada en Ortega y Gasset, discute la responsabilidad de la razón discursiva (principalmente cartesiana) en la génesis de la mentalidad y cultura secular (Cfr. Ortega y Gasset, 1985: 160-161). Escribe la malagueña:

La vida instalada en el lugar del conocimiento resulta al propio tiempo sometida a él y deificada. El primer aspecto fue denunciado por Ortega y Gasset en su crítica al idealismo desde su 'Tesis metafísica acerca de la razón vital', en las lecciones que tuve la fortuna de escucharle, cuando esto era posible, en la Facultad de Filosofía y letras de Madrid (Zambrano, 1993: 21).

Si bien entiendo que el rango axiológico de la dimensión intersubjetiva ocurre al modo como sucede en la perspectiva filosófico-vital, ella supera tal perspectiva por, como dice Cerezo Galán: "la necesidad de buscar el ser, que se echa en falta, el yo originario o el rostro del yo antes del nacimiento para hacerlo nacer y acogerlo en la vida" (2005: 33), vale decir, se trataría con Zambrano, entonces, de una intersubjetividad que habla de vida, de una que:

se desprende de la propia naturaleza de los participantes de un diálogo que ocurre en un universo constituido por Dios y el hombre. [...] que trae consigo la conciencia por parte del sujeto -y por la que se define persona- de lo que se entiende por verdadera realidad (Pulgar, 2009: 87).

Si bien la diferencia con Ortega y Gasett, se establece al momento que se comprende el significado y alcance de la crisis en las creencias, con Zambrano se avance un paso más. De suyo, la crisis en las creencias no es lo que debe importar en una hermenéutica sobre el significado de la crisis en cuanto tal, es decir, como asunto de existencia religiosa (lo que no implica dejar de tenerla presente), lo que realmente importa, es la confianza en el trascender como soporte de toda cuestión 
vital. Cuando esto falla -nos diría Zambrano- tenemos la verdadera crisis. Esta hipótesis se sostiene en que para la filósofa: "La naturaleza del alma humana, pues, está precisamente en su parentesco con lo que es divino e inmortal" (Zambrano, 2001: 51); afirmación la suya, emparentada con lo que enseńa Platón, del cual cita aquello de: "Su amor a la verdad, qué tratos apetece, en virtud de su parentesco con lo que es divino, inmortal y eterno" (Zambrano, 2001: 51) ${ }^{1}$. En efecto, al desprenderse del tutelaje de Ortega y Gasset, puede concluir que la persona ha de construir su vida - paso a paso de forma intermitente- desde el pensar la vida como algo que depende de otro. Pero este otro, se sub-entiende, es Dios.

El asunto que enfrentarse a la razón moderna para una revisión de lo religioso, y en relación a su significación para la persona humana, implica asumir la condición filosófica que la razón cartesiana pone e instala en el sujeto. De ahí que el primer paso sea la crítica al tiempo secular y, por sobre todo, a la razón moderna que es su causa y, por lo tanto, a su efecto ético entendido como un fenómeno de desolación por la cual pasa la persona a raíz del ahogo de Dios; ahogo que significa su propia agonía.

Ciertamente en Zambrano la crítica nace como reacción a la situación de la persona moderna post-cartesiana y más aun post-hegeliana (cfr., Isabel Balsa, 2000, 20 y ss.) la afirmación del sentido de la crítica es en base a una afirmación que Zambrano hace en su texto Pensamiento y poesía en la vida española. Texto en el cual la filósofa engloba la tradición filosófica que va de Parménides a Hegel bajo el denominador común de racionalismo:

Hay, pues, un horizonte amplio que se tiende desde Grecia -la Grecia parmenidiana- a la Europa de Hegel, bajo el cual toda persona, ha sido racionalista con un racionalismo fundamental que podía, inclusive, escindirse en teorías o 'ismos' de enunciación opuestas (Zambrano, 1987: 15).

Enunciada la línea de tiempo del racionalismo y sus consecuencias, Zambrano hará el ejercicio de distinguir sus efectos en la cultura y por defecto en el sujeto. Efecto que identifica como desamparo:

Pero si ha sido posible llegar a formular esta desolada y estéril definición que significa el suicidio de toda la cultura occidental, es por algo muy grave. Por una situación de desamparo, porque el hombre ha perdido el apoyo de aquellos principios que le elevaban por encima de la simple naturaleza (Zambrano, 1989: 113).

\footnotetext{
${ }^{1}$ La referencia a Platón es La República, L. X 611 a.b.
} 
El asunto que el desamparo catalogada como enfermedad moderna, a mi entender es una paradoja ética. Paradoja identificable en aquel sujeto que ve en la razón el artículo que define su esencia, es decir: "creyente en la razón como único medio de relacionarse con la realidad -razón discursiva o intuición intelectual-" (Zambrano, 1993: 196), y cuyo resultado es sentir en-sí-mismo el ahogo de Dios; ahogo vital por la desacralización merced a la razón moderna.

El postulado de la desesperanza como paradoja ética, se explica desde una filosofía construida y sostenida en una tradición del pensar español más abocado a las cuestiones de índole moral, social, política y poética. En el caso de Zambrano, este pensar característico de lo hispánico-filosófico, se elabora dinámicamente como un cuestionamiento a la modernidad desde un punto de vista crítico. Por ejemplo, los efectos desacralizadores de la razón moderna, son descritos en La Reforma del entendimiento a partir de dos claves interpretativas (Cfr, Balsa, 2000) cuyo sentido es claramente ético-religioso: 1) La razón estática y 2) La crítica a la subjetivación del sujeto cartesiano que deriva en la clausura del sujeto. Para llegar a esta conclusión, Zambrano hace uso de una metodología de análisis que incluye: 1) Los representados por Parménides en la filosofía griega y 2) Descartes en la filosofía moderna. No me detendré en el primer punto de su análisis, pues interesa más la crítica a la modernidad cartesiana. Sobre el segundo punto, Zambrano en Los Intelectuales y el drama de España, explica el modo cartesiano de enfrentar la reforma, y que metodológicamente consiste en: "volverse hacia el instrumento mismo, analizar su estructura y su funcionamiento 'puro', es decir, con independencia del objeto de aplicación. Es lo que ha hecho la crítica del idealismo moderno contra el realismo grecomedieval" (1986: 73-74).

Más allá del detalle explícito hecho a la filosofía de Descartes, tanto en el primer como en el segundo momento interpretativo, distingue en el intento racionalista (cartesiano) de reformar el entendimiento un ejercicio focalizado en su objeto de reforma (Cfr. Balsa, 2000), pues observa que es solo el instrumento -en sentido estricto: la razón- a lo que se dirige su interés, lo cual implica dejar de considerar el objeto al que la razón ha de atender como su preocupación principal.

Hecho el descubrimiento, entonces Zambrano hará presente que la reforma se debe dirigir también al objeto de la razón. El asunto que si el objeto de razón que enfrenta es el sujeto de razón que es la persona en su humanidad, para lo secular la presencia de una categoría con notas de sacralidad implica una contradicción efectiva si la razón acepta como existente aquella realidad que, en su comprensión como marco referencial, elimina referencias consideradas principales para la configuración de una moralidad que traduce aquel significado en un asunto verdaderamente humano, es decir: relación con referencias a la sacralidad que constituye existencialmente al sujeto creyente, pero que se ha visto afectado, desde la época moderna en adelante, por la fractura en los marcos de referencias u horizontes de sentido (Cfr. Taylor, 2006). 
Se puede concluir a partir del juicio indicado sobre la fractura de los marcos de referencia, que la situación moderna es ciertamente paradojal; a causa que la explicación sobre el sentido de la realidad que el sujeto elabora para sí y para otros como propuesta de significado, descansa en una razón presa de su propia condición, vale decir, de su limitación intelectiva cuando se trata de Dios, y que es el sentido de la frase citada antes sobre el drama de la razón (Cfr. Hacia un saber sobre el alma). Sin embargo, y curiosamente, la limitación de la razón es la que lleva a que, por variables que interpretamos son propias de la secularización (al menos en occidente), se deba reconocer que:

Las experiencias, formas, plasmaciones e ideas religiosas de la humanidad son infinitamente ricas, e infinitamente compleja en su problemática.

El simple intento de describir [...] las diferentes concepciones de Dios constituiría una osadía: resulta absolutamente imposible abarcar sus divergencias, sus diferenciaciones e implicaciones (Küng, 1979: 643).

La tesis de Küng se enriquece y se explica -además- por la variación de la experiencia religiosa, o del modo como se acepta, se asume y se comprende por parte de los sujetos a lo largo del tiempo y en espacios diversos. Creo que Zambrano es una muestra de esfuerzo filosófico por resolver este aspecto evolutivo de la experiencia religiosa montada en la paradoja de lo variable, de lo que aparece y desaparece de tanto en tanto como lo declara en el libro III Los procesos de lo divino de El hombre y lo divino (1983: 229-317), puesto que ahí describe e interpreta el cambio de influencia onto-teológica que se da entre lo medieval y lo moderno. Descripción que la acerca a Unamuno, signo además de la influencia del pensador vasco en Zambrano. No es menor que Unamuno llegue al punto de jugar con la paradoja de la "Disolución racional" para explicar lo que ocurre en el sujeto por gracia del imperio de la razón moderna. Véase a propósito de esto, el reclamo unamuniano en lo que sigue:

Es una cosa terrible la inteligencia. Tiende a la muerte como a la estabilidad la memoria. Lo vivo, lo que es absolutamente inestable, lo absolutamente individual es, en rigor, ininteligible. La lógica tiende a reducirlo todo a entidades y a géneros, a que no tenga cada representación más que un solo y mismo contenido en cualquier lugar, tiempo o relación en que se nos ocurra. Y no hay nada nada que no sea lo mismo en los momentos sucesivos de su ser (Unamuno, 1983: 140).

Aparcando a Unamuno, pero manteniendo la arquitectura crítica de Zambrano, retomamos señalando que si en la Edad Media el sentido era religioso -sentido observado en la inteligencia tenida por parte de los sujetos sobre la realidad bajo el apelativo de la fe en procura de entregar certeza de bien- en la Edad 
Moderna se inicia el proceso de ocultamiento de la fe como fuente de comprensión del significante del bien (Cfr., Taylor, 2006). Este ocultamiento -siguiendo aquí a Taylor- llega hasta el punto de afectar la imagen del mundo por un cambio ocurrido a nivel existencial, con el agregado de las circunstancias culturales gestionadas por la razón moderna. De suyo, la cultura moderna es una arquitectura sostenida en los pilares de la razón. Acción arquitectónica que interviene como barrera a la hora de asumir la disponibilidad a lo trascendente y, por tanto, en la conquista de una comprensión de lo divino. Al respecto, Zambrano advierte sobre los límites y peligros; advertencia que se ubica dentro de su crítica-descriptiva a la razón:

La filosofía moderna, origen de la física matemática, se queda en soledad para dar una imagen del cosmos, y al desprenderse por completo de la revelación queda librada a sí misma, como quería. Y el hombre que a la filosofía o a la ciencia se acoge, aun el hombre común que respira este clima, queda librado a su soledad humana, a la soledad del género humano sin cosmos y sin revelación. Y aquellos que vivían dentro aún de una religión determinada quedan escindidos, separados del pensamiento filosófico-científico y del ambiente intelectual y moral que de ellos emana, creyendo a medias y por partida doble (2003: 12).

Visto el asunto de la limitación de la razón para una inteligencia de lo religioso, por tanto, de aquello que habita en la persona como acto esencial de ser, nos topamos que la explicación de Zambrano sobre la razón discursiva, rescata el valor significativo de una experiencia tipo que, llevada por la persona, se compromete en un juego de cambios de comprensión nacidas desde una perspectiva onto-teológica. Para poder dilucidar el sentido de este proceso, Zambrano se obliga a buscar tanto en la persona como en el hecho que lo divino se caracteriza por ser:

lo irreductible a lo humano, configurado de diversas maneras según sean los aspectos que eso divino haya tomado, según sean los afanes y anhelos del hombre. Y en cualquiera de los casos ha llegado el instante terrible de que 'eso divino' irreductible a lo humano ha corrido la suerte de lo humano: pasar, ser vencido y aún morir (1993: 136).

El cambio de paradigma del horizonte de comprensión, termina por dar vida a una mirada humana fuertemente condicionada por una modernidad que hizo de la secularización su caballo de batalla (Cfr., Farre, 1969: 431 y ss), y cuyo resultado es una transformación de la figura religiosa en incordio o incomodidad existencial (González de Cardedal, 2004: 68); cuestión evidente mientras más se avanza bajo el paraguas del progreso, pues como advierte Küng: 
¿Cómo debe el hombre en realidad, en la realidad una y pluridimensional, pensar a Dios? Esta pregunta, en torno a lo cual giró siempre la filosofía, se ha hecho cada vez más acuciante desde Descarte y Pascal.

[...] hoy en día, tanto si se opta por Dios como si se opta contra él, la opción debe hacerse en concreto teniendo presente no tanto la comprensión de Dios griega o medieval cuanto la comprensión moderna de Dios (2005: 159).

Es probable que la pregunta que introduce el punto B de su texto, responda al hecho constatado de seguir siendo Dios aguijón y dilema en la afirmación que hacemos del universo, pero también de su negación crítica y su transformación que, se quiera o no, nos cambia dinámicamente.

\section{CONCLUSIÓN}

Zambrano pertenece al grupo de intelectuales que tuvo conciencia clara de la inevitable crisis de la mentalidad burguesa en la sociedad liberal al filo del siglo XX, así como de las repercusiones que dicha crisis tuvo en el campo de las ideas filosóficas y políticas (Cfr., Bungard, 2000). Esta conciencia se visualiza en el campo de las descripciones del sujeto religioso en ambientes secularizados y sus efectos en el campo de la vida humana y, por cierto, cultural. Ejercicio filosófico que dialécticamente la emparenta con Ortega y Gasset y al mismo tiempo la distancia de él.

Hoy en día existe coincidencia entre los lectores de Zambrano que hay una línea de lecturas e influencias filosóficas que parte con Ortega y Gasset a la hora de hacer la crítica a la razón moderna, pero que luego se ve enriquecida por otras influencias entre las cuales, según Pedro Cerezo Galán (2005) se encuentra Unamuno, Zubiri, Azorín. En esta línea Bungard dice que hay otras influencias que intervienen en la génesis y maduración su pensamiento. Esta idea acaba por ser una evidencia que no contradice la originalidad de Zambrano, pues no pierde carácter de obra única sin dejar por ello de mantener relación de afinidad con otros que la anteceden en el espacio cultural español:

el pensamiento zambraniano en lo que tiene de filosofía política y de análisis crítico de la cultura ofrece más afinidades con el pensamiento de escritores como Ángel Gavinet, Miguel de Unamuno, Valle-Inclán o Antonio Machado que con el pensamiento europeísta, racional, 'luminoso' y optimista de su maestro Ortega y Gasset (Bungard, 2000: 33).

Más de todos los que influyen en Zambrano en el desarrollo de una razón capaz de responder afirmativamente a la religiosidad desde un enfoque optimista 
-éticamente esperanzador-, me quedo con reconocer las influencias pero no para insistir en ellas al punto de perder lo propiamente original de Zambrano, pues creo que si bien:

Su filosofía es de crisis y, a pesar de la desolación de la cual arranca su pensamiento, es también filosofía de esperanza, de una esperanza que sostiene la existencia negándola, es decir, remitiéndola a su fundamento en la nada, en el no-ser, aunque sin angustia existencial, sin náusea existencialista, sin nihilismo, con una firme esperanza (2000: 38).

Con todo, y aceptando el peso de la influencia de figuras del pensamiento hispánico en Zambrano, hay pruebas suficientes del carácter único del pensamiento de la malagueña en el campo de la reflexión crítica, particularmente cuando de la razón moderna en su vertiente cartesiana se trata. Mas para no quedar atrapada en la crítica, sino para visualizar una propuesta de respuesta a la angustia que provoca en el sujeto la tendencia a la absolutización de la razón - "La cultura moderna fue arrojada de sí al ser total del hombre, cuidándose sólo de su pensamiento" (Zambrano, 1989: 22)-Zambrano discute en Hacia un saber sobre el alma la razón del racionalismo. Según su lectura, esta al quedar atrapada en el pensamiento del sujeto y sus leyes, se encontró incapacitada para pensar el alma y dejó la totalidad del sujeto como terreno que ya no le competía, con lo cual al renunciar aquello: "el hombre pasó a ser objeto de ciencias que no eran Filosofía" (García, 2005: 22). A mi parecer, la crítica de Zambrano descubre en su esencia la anulación del sujeto, por tanto, la desesperanza. De ahí que comprendida la desesperanza y la desolación que ella genera, se obligue a buscar en la religiosidad la vía para aliviar del ahogo a la existencia por exceso de razón discursiva, ofreciendo una razón que, siendo poética, es tanto vía de pregunta como vía de respuesta. Respuestas, eso sí, que superan con facilidad el simple uso de la razón científica, pues su propuesta es la razón poética, forma de razón que tributa a una comprensión de lo ético enganchado en lo sagrado.

En suma, Zambrano es capaz -desde una descripción de la composición de la razón moderna- significar el sentido de la desolación o desamparo, como efecto del imperio de la razón cartesiana en la construcción del sujeto moderno, pero, además, comprendido el asunto, se atreve a ofrecer una respuesta filosófica cuyo núcleo de acción es la resignificación de la esperanza; la cual nace de la necesidad de superar el sentido negativo que tiene para el sujeto la modernidad (Cfr. Bungard, 2005: 38 ss). Superación posible al recuperar el sentido de la tierra como lugar de vínculo que sostiene las creencias: 
Es en lo negativo donde la esperanza encuentra su campo, su lugar, cuando simbólica o realmente la vida falta, la tierra es el lugar que nos sostiene. El símbolo de la tierra abarca todo aquello que continuamente nos sostiene, sin que nos demos mucha cuenta fuera de nosotros y dentro de ese 'contar con' que según Ortega y Gasset es el referimiento continuo a lo que está ahí sin más, lo que forma el estrato de los supuestos [...] que están depositados en las creencias sobre las cuales se alzan las ideas (Zambrano, 1991: 102).

Cuestión posible si el sujeto en cuanto persona es "llevado por la esperanza" (Zambrano, 1991: 103) al momento que emprende el viaje de su propia humanización.

\section{REFERENCIAS}

Balsa, Isabel. Tiempo y escritura en María Zambrano. Donostía: Iralka, 2000.

Bungard, Ana. Más allá de la filosofía. Sobre el pensamiento filosófico-mistico de María Zambrano. Madrid: Trotta, 2000.

Cerezo Galán, Pedro. La herencia de M. de Unamuno, J. Ortega y Gasset y X. Zubiri en María Zambrano. En J. L. Mora García y J. M. Moreno Yuste (Eds.), Pensamiento y palabra en recuerdo de María Zambrano 1904-1991. España: Junta de Castilla y León, (2005): 19-50.

Cobos Navidad, María Nieves. Recuperar lo divino en el hombre. Reflexión en torno a la obra de María Zambrano 'El hombre y lo divino’. En I. Murillo Murillo (Ed.), Dios, hombre, praxis. Madrid: Diálogo Filosófico, (1998): 161-176.

Cortina, Adela. Ética minima, Madrid: Tecnos, 1986.

Farre, Luis. Filosofía de la religión. Buenos Aires: Losada, 1969.

García, Juan José. Persona y contexto socio-histórico en Maria Zambrano. Cuadernos de Pensamiento Espańol 28. Pamplona: Servicio de Publicaciones de la Universidad de Navarra, 2005.

Gómez Blesa, Mercedes. Prólogo a Claros del bosque. Madrid: Cátedra, 2011. 
González de Cardedal, Olegario. Dios. Salamanca: Sígueme, 2004.

Küng, Hans. ¿Existe Dios? Madrid: Trotta, 1979.

Ortega y Gasset, José. ¿Qué es filosofia? Madrid: Alianza, 1985.

Pulgar, Rodrigo. Sentido y significado de la oblación para la constitución del sujeto. Revista Diálogo Filosófico, No 73, septiembre-octubre, Madrid, (2009): 8595.

Taylor, Charles. Las fuentes del yo. La construcción de la identidad Moderna. Barcelona: Paidos, 2006.

Unamuno, Miguel de. Del sentimiento trágico de la vida. Madrid: AKAL, 1983.

Velasco, Juan Martín. Introducción a la fenomenología de la religión. Madrid: Trotta, 2006.

Zambrano, María. Los intelectuales y el drama de España. Barcelona: Anthropos, 1986.

Zambrano, Zambrano, María. La reforma del entendimiento. Revista Atenea, Año XIV, Tomo XXXVII, Nº 139, Concepción, (1937): 115-124.

Zambrano, María. Pensamiento y poesía en la vida española. Madrid: Endymion, 1987.

- Hacia un saber sobre el alma. Madrid: Alianza, 1989.

El hombre y lo divino. Madrid: FCE, 1993.

- Filosofia y Poesía. Madrid: FCE, 2001.

- Unamuno. Barcelona: Debate, 2003. 